FACTA UNIVERSITATIS (NIŠ)

Ser. Math. Inform. Vol. 36, No 1 (2021), 179-189

https://doi.org/10.22190/FUMI200804015G

Original Scientific Paper

\title{
PROPERTIES OF A NEW SUBCLASS OF ANALYTIC FUNCTION ASSOCIATED TO RAFID - OPERATOR AND $q$-DERIVATIVE
}

\author{
Mohammad Hassn Golmohammadi and Shahram Najafzadeh \\ Faculty of Mathematical Sciences, Department of Pure Mathematics, \\ Payame Noor University, P. O. Bax: 19395 - 3697, Tehran, Iran
}

\begin{abstract}
In this article, we introduce a new subclass of analytic functions, using the exponent operators of Rafid and $q$-derivative. The coefficient estimates, extreme points, convex linear combination, radii of starlikeness, convexity and finally integral have been investigated.
\end{abstract}

Keywords: Rafid - operator, $q$-derivative, $q$-integral, univalent function, coefficient bound, convex set, partial sum.

\section{Introduction}

The theory of univalent functions can be described by using the theory of the $q$ calculus. In recent years, such $q$-calculus as the $q$-integral and $q$-derivative have been used to construct several subclasses of analytic functions [1, 6, 11, 12]. The theory of $q$-analysis has motivated the researchers owing to many branches of mathematics and physics. For example, in the areas of special functions, $q$-difference, $q$-integral equations, optimal control problems, $q$-difference, $q$-integral equations, $q$-transform analysis and in quantum physics see for instance, [7, 8, 10, 14].

The main subject of the present paper is to introduce and investigate a new subclass of analytic functions in the open unit disk $U$ by using the operators Rafid and $q$-derivative. Let $\mathcal{A}$ denote the class of functions $f(z)$ in the form of:

$$
f(z)=z+\sum_{k=2}^{+\infty} a_{k} z^{k}
$$

Received August 4, 2020; accepted November 26, 2020.

Corresponding Author: Mohammad Hassan Golmohammadi, Faculty of Mathematical Sciences, Department of Pure Mathematics, Payame Noor University, P. O. Bax: 19395 - 3697, Tehran, Iran | E-mail: golmohamadi@pnu.ac.ir

2010 Mathematics Subject Classification. Primary 20D15; Secondary 20F14

ㄷ 2021 by University of Niš, Serbia | Creative Commons License: CC BY-NC-ND 
which are analytic in the punctured unit disk

$$
U=\{z \in \mathbb{C}:|z|<1\} .
$$

For $f(z) \in \mathcal{A}$, the $q$ - derivative, $0<q<1$, of $f(z)$ is defined by Gasper and Rahman $[5]$.

$$
D_{q} f(z)= \begin{cases}\frac{f(q z)-f(z)}{(q-1) z} & (z \neq 0) \\ f^{\prime}(0) & (z=0) .\end{cases}
$$

where $z \in U$ and $0<q<1$.

Let $T(p)$ be the class of all $p$-valent functions of the form

$$
f(z)=z^{p}-\sum_{n=p+1}^{+\infty} a_{n} z^{n} \quad a_{n} \geq 0,
$$

which are analytic in the punctured unit disk

$$
U=\{z \in \mathbb{C}:|z|<1\} .
$$

If $f \in T(p)$ is given by Equation (1.3) and $g \in T(p)$ is given by

$$
g(z)=z^{p}-\sum_{n=p+1}^{+\infty} b_{n} z^{n} \quad b_{n} \geq 0,
$$

then the Hadamard product $f * g$ of $f$ and $g$ is defined by

$$
(f * g)(z)=z^{p}-\sum_{n=p+1}^{+\infty} a_{n} b_{n} z^{n}=(g * f)(z) .
$$

From Equation (1.2) for a function $f(z)$ given by Equation(1.3) we get

$$
D_{q} f(z)=[p]_{q} z^{p-1}-\sum_{n=p+1}^{\infty}[n]_{q} a_{n} z^{p-1} \quad, \quad z \in U
$$

where

$$
[p]_{q}:=\frac{1-q^{p}}{1-q}=1+q+q^{2}+\cdots+q^{p-1}
$$

and

$$
[n]_{q}:=\frac{1-q^{n}}{1-q}=1+q+q^{2}+\cdots+q^{n-1} .
$$

Also $[p]_{q} \rightarrow p$ and $[n]_{q} \rightarrow n$ as $q \rightarrow \overline{1}$. So we conclude that

$$
\lim _{q \rightarrow \overline{1}} D_{q} f(z)=f^{\prime}(z) \quad, \quad z \in U
$$


see also [13].

Waggas and Rafid defined the Rafid -operator of a function $f(z)=z-\sum_{n=2}^{+\infty} a_{n} z^{n}$ by

$$
R_{\mu}^{\theta}(f(z))=z-\sum_{n=2}^{+\infty} \frac{(1-\mu)^{n-1} \Gamma(\theta, n)}{\Gamma(\theta+1)} a_{n} z^{n} .
$$

See for instance, $[2,3,4])$.

By using Rafid and $q$-derivative operators, we define the $R_{\mu}^{\theta} D_{q}(f(z))$ for a function $f \in T(p)$ as follows:

Definition 1.1. The Rafid-operator of $f \in T(p)$, is denoted by $R_{\mu}^{\theta} D_{q}$ and defined as following:

$$
R_{\mu}^{\theta} D_{q}(f(z))=\frac{z}{[p]_{q}(1-\mu)^{p+\theta+1} \Gamma(p+\theta+1)} \int_{0}^{+\infty} t^{\theta-1} e^{-\left(\frac{t}{1-\mu}\right)} D_{q}(f(z t)) d t
$$

Then it is easy to deduce the series representation of the function $R_{\mu}^{\theta}(f(z))$ as following:

$$
\begin{aligned}
R_{\mu}^{\theta} D_{q}(f(z)) & =z^{p}-\sum_{n=p+1}^{+\infty} \frac{[n]_{q}(1-\mu)^{n-p} \Gamma(n+\theta+1)}{[p]_{q} \Gamma(p+\theta+1)} a_{n} z^{n} \\
& =z^{p}-\sum_{n=p+1}^{+\infty} M(n, p, q, \mu, \theta) a_{n} z^{n}
\end{aligned}
$$

where

$$
M(n, p, q, \mu, \theta)=\frac{[n]_{q}(1-\mu)^{n-p} \Gamma(n+\theta+1)}{[p]_{q} \Gamma(p+\theta+1)} .
$$

We now define a new subclass $T_{p, q} R(\lambda, \alpha, \beta, \mu, \theta)$ of analytic functions of $T(p)$ by using the operators Rafid and $q$-derivative. Let $f(z) \in T(p)$ is said to be in the class $T_{p, q} R(\lambda, \alpha, \beta, \mu, \theta)$ if and only if it satisfies the inequality:

$$
\left|\frac{\lambda z^{2}\left(R_{\mu}^{\theta}\left(D_{q}(f * g)(z)\right)\right)^{\prime \prime}+z\left(R_{\mu}^{\theta}\left(D_{q}(f * g)(z)\right)\right)^{\prime}}{z\left(R_{\mu}^{\theta}\left(D_{q}(f * g)(z)\right)\right)^{\prime}+(1-\lambda)\left(R_{\mu}^{\theta}\left(D_{q}(f * g)(z)\right)\right)}-(1-\beta)\right| \leq \alpha .
$$

Here, $0<q<1,0 \leq \lambda<1,0 \leq \alpha \leq 1,0 \leq \mu<1,0 \leq \theta \leq 1$ and $\beta<1$.

\section{Main Results}

Unless otherwise mentioned, we suppose throughout this paper that $0<q<1,0 \leq$ $\lambda<1,0 \leq \alpha \leq 1,0 \leq \mu<1,0 \leq \theta \leq 1$ and $\beta<1$. First we state coefficient estimates on the class $T_{p, q} R(\lambda, \alpha, \beta, \mu, \theta)$. 
Theorem 2.1. Let $f(z) \in T(p)$, then $f(z) \in T_{p, q} R(\lambda, \alpha, \beta, \mu, \theta)$ if and only if (2.1) $\sum_{n=p+1}^{+\infty}[(n(n-1)-(\alpha+\beta)+1) \lambda+(n+1)(\alpha+\beta)-1] M(n, p, q, \mu, \theta) a_{n} b_{n} \leq 1-2 \lambda$.

Proof. Suppose $f(z)$ difined by Equation(1.3) and $f(z) \in T_{p, q} R(\lambda, \alpha, \beta, \mu, \theta)$, then Equation (1.11) holds true, we have

$$
\begin{gathered}
\mid \frac{[(2-\beta) \lambda+2 \beta-1] z^{p}}{(2-\lambda) z^{p}-(n-\lambda+1) \sum_{n=p+1}^{+\infty} M(n, p, q, \mu, \theta) a_{n} b_{n} z^{n}} \\
-\frac{[n(1-n)+\beta-1] \lambda+1-(1+n) \beta] \sum_{n=p+1}^{+\infty} n(n-1) M(n, p, q, \mu, \theta) a_{n} b_{n} z^{n}}{(2-\lambda) z^{p}-(n-\lambda+1) \sum_{n=p+1}^{+\infty} M(n, p, q, \mu, \theta) a_{n} b_{n} z^{n}} \mid<\alpha .
\end{gathered}
$$

Since $\operatorname{Re}(z) \leq|z|$ for all $z$,

$\operatorname{Re}\left\{\frac{[(2-\beta) \lambda+2 \beta-1] z^{p}}{(2-\lambda) z^{p}-(n-\lambda+1) \sum_{n=p+1}^{+\infty} M(n, p, q, \mu, \theta) a_{n} b_{n} z^{n}}\right.$
$\left.-\frac{[n(1-n)+\beta-1] \lambda+1-(1+n) \beta] \sum_{n=p+1}^{+\infty} n(n-1) M(n, p, q, \mu, \theta) a_{n} b_{n} z^{n}}{(2-\lambda) z^{p}-(n-\lambda+1) \sum_{n=p+1}^{+\infty} M(n, p, q, \mu, \theta) a_{n} b_{n} z^{n}}\right\}<\alpha$.

By letting $z \rightarrow \overline{1}$ through real values, we have

$\sum_{n=p+1}^{+\infty}[(n(n-1)-(\alpha+\beta)+1) \lambda+(n+1)(\alpha+\beta)-1] M(n, p, q, \mu, \theta) a_{n} b_{n} \leq 1-2 \lambda$.

Conversely, let Equation (2.1) holds true, it is enough to show that

$X(f)=\mid \lambda z^{2}\left(R_{\mu}^{\theta}\left(D_{q}(f * g)(z)\right)\right)^{\prime \prime}+z\left(R_{\mu}^{\theta}\left(D_{q}(f * g)(z)\right)\right)^{\prime}$

$-(1-\beta)\left[z\left(R_{\mu}^{\theta}\left(D_{q}(f * g)(z)\right)\right)^{\prime}+(1-\lambda)\left(R_{\mu}^{\theta}\left(D_{q}(f * g)(z)\right)\right)\right] \mid$

$-\alpha\left|z\left(R_{\mu}^{\theta}\left(D_{q}(f * g)(z)\right)\right)^{\prime}+(1-\lambda)\left(R_{\mu}^{\theta}\left(D_{q}(f * g)(z)\right)\right)\right| \leq 0$

But for $0<|z|=r<1$ we have

$$
\begin{aligned}
& X(f)=\mid\left[\left(2-\beta \lambda\left[z^{p}-\sum_{n=p+1}^{+\infty} n(n-1) M(n, p, q, \mu, \theta) a_{n} b_{n} z^{n}\right]\right.\right. \\
& +z^{p}-\sum_{n=p+1}^{+\infty} n M(n, p, q, \mu, \theta) a_{n} b_{n} z^{n} \\
& -(1-\beta)\left(\left[z^{p}-\sum_{n=p+1}^{+\infty} n M(n, p, q, \mu, \theta) a_{n} b_{n} z^{n}\right]\right. \\
& \left.+(1-\lambda)\left[z^{p}-\sum_{n=p+1}^{+\infty} n M(n, p, q, \mu, \theta) a_{n} b_{n} z^{n}\right]\right) \mid \\
& -\alpha\left(\left[z^{p}-\sum_{n=p+1}^{+\infty} n M(n, p, q, \mu, \theta) a_{n} b_{n} z^{n}\right]\right. \\
& \left.+(1-\lambda)\left[z^{p}-\sum_{n=p+1}^{+\infty} n M(n, p, q, \mu, \theta) a_{n} b_{n} z^{n}\right]\right) \mid \\
& \leq \sum_{n=p+1}^{+\infty}[(n(n-1)-(\alpha+\beta)+1) \lambda+(n+1)(\alpha+\beta)-1] M(n, p, q, \mu, \theta)\left|a_{n}\right|\left|b_{n}\right| r^{n} \\
& -(1-2 \lambda) .
\end{aligned}
$$


Since the above inequality holds for all $r(0<r<1)$, by letting $r \rightarrow \overline{1}$ and using Equation(2.1) we obtain $X(f) \leq 0$. This completes the proof.

Corollary 2.1. If function $f(z)$ of the form Equation (1.3) belongs to $T_{p, q} R(\lambda, \alpha, \beta, \mu, \theta)$ then

$$
a_{n} \leq \frac{1-2 \lambda}{[(n(n-1)-(\alpha+\beta)+1) \lambda+(n+1)(\alpha+\beta)-1] M(n, p, q, \mu, \theta) b_{n}}
$$

where

$$
M(n, p, q, \mu, \theta)=\frac{[n]_{q}(1-\mu)^{n-p} \Gamma(n+\theta+1)}{[p]_{q} \Gamma(p+\theta+1)}, \quad n \geq p+1 .
$$

With the equality for the function

$$
f(z)=z^{p}-\frac{1-2 \lambda}{[(n(n-1)-(\alpha+\beta)+1) \lambda+(n+1)(\alpha+\beta)-1] M(n, p, q, \mu, \theta) b_{n}} z^{p}
$$

Next we obtain extreme points and convex linear combination property for $f(z)$ belongs to $T_{p, q} R(\lambda, \alpha, \beta, \mu, \theta)$.

Theorem 2.2. The function $f(z)$ of the form Equation (1.3) belongs to $T_{p, q} R(\lambda, \alpha, \beta, \mu, \theta)$ if and only if it can be expressed by

$$
f(z)=\sigma_{1} f_{1}(z)+\sum_{n=p+1}^{\infty} \sigma_{n} f_{n}(z), \quad \sigma_{n} \geq 1, \quad \sigma_{1}+\sum_{n=p+1}^{\infty} \sigma_{n}=1
$$

where

$f_{1}(z)=z^{p}$

$f_{n}(z)=\frac{1-2 \lambda}{[(n(n-1)-(\alpha+\beta)+1) \lambda+(n+1)(\alpha+\beta)-1] M(n, p, q, \mu, \theta) b_{n}} z^{k}$,

$$
(n \geq p+1) \text {. }
$$

Proof. Let

$$
\begin{aligned}
f(z) & =\sigma_{1} f_{1}(z)+\sum_{n=p+1}^{\infty} \sigma_{n} f_{n}(z) \\
& =\sigma_{1} f_{1}(z)+\sum_{n=p+1}^{\infty} \sigma_{n}\left[z^{n}-\frac{1-2 \lambda}{[(n(n-1)-(\alpha+\beta)+1) \lambda+(n+1)(\alpha+\beta)-1] M(n, p, q, \mu, \theta) b_{n}}\right] z^{n} \\
& =z^{p}-\sum_{n=p+1}^{\infty} \frac{1-2 \lambda}{[(n(n-1)-(\alpha+\beta)+1) \lambda+(n+1)(\alpha+\beta)-1] M(n, p, q, \mu, \theta) b_{n}} \sigma_{n} z^{n} .
\end{aligned}
$$


Now apply Theorem 2.1 to conclude that $f(z) \in T_{p, q} R(\lambda, \alpha, \beta, \mu, \theta)$. Conversely, if $f(z)$ given by Equation (1.3) belongs to $T_{p, q} R(\lambda, \alpha, \beta, \mu, \theta)$, by letting

$$
\sigma_{1}=1-\sum_{n=p+1}^{+\infty} \sigma_{n}
$$

where

$$
\sigma_{k}=\frac{[(n(n-1)-(\alpha+\beta)+1) \lambda+(n+1)(\alpha+\beta)-1] M(n, p, q, \mu, \theta) b_{n}}{1-2 \lambda} a_{n}
$$

we conclude the required result.

Theorem 2.3. Let for $t=1,2, \cdots, k, f_{t}(z)=z^{p}-\sum_{n=p+1}^{+\infty} a_{n, t} z^{n}$ belongs to $T_{p, q} R(\lambda, \alpha, \beta, \mu, \theta)$, then $F(z)=\sum_{t=1}^{k} \sigma_{t} f_{t}(z)$ is also in the same class, where $\sum_{t=1}^{k} \sigma_{t}=1$. Hence $T_{p, q} R(\lambda, \alpha, \beta, \mu, \theta)$ is a convex set.

Proof. According to Theorem 2.1 for every $t=1,2, \cdots, k$ we have

$$
\sum_{n=p+1}^{+\infty}[(n(n-1)-(\alpha+\beta)+1) \lambda+(n+1)(\alpha+\beta)-1] M(n, p, q, \mu, \theta) a_{n, t} b_{n} \leq 1-2 \lambda
$$

But

$$
\begin{aligned}
F(z) & =\sum_{t=1}^{k} \sigma_{t} f_{t}(z) \\
& =\sum_{t=1}^{k} \sigma_{t}\left(z^{p}-\sum_{n=p+1}^{\infty} a_{n, t} z^{n}\right) \\
& =z^{p} \sum_{t=1}^{k} \sigma_{t}-\sum_{n=p+1}^{\infty}\left(\sum_{t=1}^{k} \sigma_{t} a_{n, t}\right) z^{n} \\
& =z^{p}-\sum_{n=p+1}^{\infty}\left(\sum_{t=1}^{k} \sigma_{t} a_{n, t}\right) z^{n} .
\end{aligned}
$$


Since

$$
\begin{aligned}
& \sum_{n=p+1}^{+\infty}[(n(n-1)-(\alpha+\beta)+1) \lambda+(n+1)(\alpha+\beta)-1] \\
& \times M(n, p, q, \mu, \theta) a_{n, t} b_{n}\left(\sum_{n=1}^{m} \sigma_{n} a_{k, n}\right) \\
= & \sum_{n=1}^{m} \sigma_{n}\left(\sum_{n=p+1}^{+\infty}[(n(n-1)-(\alpha+\beta)+1) \lambda+(n+1)(\alpha+\beta)-1]\right. \\
& \times M(n, p, q, \mu, \theta) a_{n, t} b_{n} \\
\leq & \sum_{t=1}^{k} \sigma_{t}(1-2 \lambda)=(1-2 \lambda) \sum_{t=1}^{k} \sigma_{t}=1-2 \lambda,
\end{aligned}
$$

by Theorem 2.1 the proof is complete.

\section{Radii of close-to-convexity, starlikeness and convexity}

In this section we obtain radii of close-to-convexity, starlikeness, convexity and investigate about partial sum property.

In the proof of next theorem, we need the Alexander's Theorem. This theorem states that if $f$ is an analytic function in the unit disk and normalized by $f(0)=$ $f^{\prime}(0)-1=0$, then $f(z)$ is convex if and only if $z f^{\prime}(z)$ is starlike.

Theorem 3.1. Let $f(z)$ of the form Equation (1.3) belongs to $T_{p, q} R(\lambda, \alpha, \beta, \mu, \theta)$ then and

(i) $f(z)$ is $p$-valently close-to-convex of order $\gamma$ in $|z|<R_{1}$, where $0 \leq \gamma<p$

$$
R_{1}=\inf _{n}\left\{\frac{(p-\gamma)[(n(n-1)-(\alpha+\beta)+1) \lambda+(n+1)(\alpha+\beta)-1] M(n, p, q, \mu, \theta) a_{n} b_{n}}{n(1-2 \lambda)}\right\}^{\frac{1}{n-p}},
$$

(ii) $f(z)$ is $p$-valently starlike of order $\gamma$ in $|z|<R_{2}$, where $0 \leq \gamma<p$ and

$$
R_{2}=\inf _{n}\left\{\frac{(p-\gamma)[(n(n-1)-(\alpha+\beta)+1) \lambda+(n+1)(\alpha+\beta)-1] M(n, p, q, \mu, \theta) a_{n} b_{n}}{(n-\gamma)(1-2 \lambda)}\right\}^{\frac{1}{n-p}},
$$

(iii) $f(z)$ is $p$-valently convex of order $\gamma$ in $|z|<R_{3}$, where $0 \leq \gamma<p$ and

$$
R_{3}=\inf _{n}\left\{\frac{p(p-\gamma)[(n(n-1)-(\alpha+\beta)+1) \lambda+(n+1)(\alpha+\beta)-1] M(n, p, q, \mu, \theta) a_{n} b_{n}}{n(n-\gamma)(1-2 \lambda)}\right\}^{\frac{1}{n-p}} .
$$

Proof. (i) For close-to-convexity it is enough to show that

$$
\left|\frac{z f^{\prime}}{z^{p-1}}-p\right|<p-\gamma
$$


but

$$
\left|\frac{z f^{\prime}}{z^{p-1}}-p\right|=\left|\frac{p z^{p-1}-\sum_{n=p+1}^{+\infty} n a_{n}|z|^{n}-p z^{p-1}}{z^{p-1}}\right| \leq \sum_{n=p+1}^{+\infty} n a_{n}|z|^{n-p} \leq p-\gamma,
$$

or $\sum_{n=p+1}^{+\infty} \frac{n}{p-\gamma} a_{n}|z|^{n-p} \leq 1$. By using Equation (2.1) we obtain

$$
\begin{aligned}
& \sum_{n=p+1}^{+\infty} \frac{n}{p-\gamma} a_{n}|z|^{n-p} \\
& \leq \sum_{k=1}^{+\infty} \frac{n(1-2 \lambda)|z|^{n-p}}{(p-\gamma)[(n(n-1)-(\alpha+\beta)+1) \lambda+(n+1)(\alpha+\beta)-1]} \\
& \times \frac{1}{M(n, p, q, \mu, \theta) a_{n} b_{n}} \leq 1 .
\end{aligned}
$$

So, it is enough to suppose

$$
\begin{aligned}
& |z|^{n-p} \\
& \leq \frac{(p-\gamma)[(n(n-1)-(\alpha+\beta)+1) \lambda+(n+1)(\alpha+\beta)-1] M(n, p, q, \mu, \theta) a_{n} b_{n}}{n(1-2 \lambda)},
\end{aligned}
$$

which completes the case $(i)$. But

(ii) For starlikeness it is enough to show that $\left|\frac{z f^{\prime}}{f}-p\right|<p-\gamma$.

$$
\left|\frac{z f^{\prime}}{f}-p\right|=\left|\frac{\sum_{n=p+1}^{+\infty}(n-p) a_{n} z^{n}}{z^{p}-\sum_{n=p+1}^{+\infty} a_{n} z^{n}}\right| \leq \frac{\sum_{n=p+1}^{+\infty}(n-p) a_{n}|z|^{n-p}}{1-\sum_{n=p+1}^{+\infty} a_{n}|z|^{n-p}} \leq p-\gamma .
$$

Therefore,

$$
\sum_{n=p+1}^{+\infty}(n-p) a_{n}|z|^{n-p} \leq(p-\gamma)\left(1-\sum_{n=p+1}^{+\infty} a_{n}|z|^{n-p}\right),
$$

or

$$
\sum_{n=p+1}^{+\infty} \frac{n-\gamma}{p-\gamma} a_{n}|z|^{n-p} \leq 1
$$

Now by Equation (2.1), we obtain

$$
\begin{aligned}
& \sum_{n=p+1}^{+\infty} \frac{n-\gamma}{p-\gamma} a_{n}|z|^{n-p} \\
& \leq \sum_{n=p+1}^{+\infty} \frac{(n-\gamma)(1-2 \lambda)|z|^{n-p}}{(p-\gamma)[(n(n-1)-(\alpha+\beta)+1) \lambda+(n+1)(\alpha+\beta)-1]} \\
& \times \frac{1}{M(n, p, q, \mu, \theta) a_{n} b_{n}} \leq 1 .
\end{aligned}
$$


So, it is enough to suppose

$$
\begin{aligned}
& |z|^{n-p} \\
& \leq \frac{(p-\gamma)[(n(n-1)-(\alpha+\beta)+1) \lambda+(n+1)(\alpha+\beta)-1] M(n, p, q, \mu, \theta) a_{n} b_{n}}{(n-\gamma)(1-2 \lambda)} .
\end{aligned}
$$

Hence we get the required result.

(iii) For convexity, by Alexander's Theorem and by applying an easy calculation, we reach the required result. Hence the result.

Theorem 3.2. The class $T_{p, q} R(\lambda, \alpha, \beta, \mu, \theta)$ is a convex set.

Proof. Let $f(z)=z^{p}-\sum_{n=p+1}^{+\infty} a_{n} z^{n}$ and $g(z)=z^{p}-\sum_{n=p+1}^{+\infty} b_{n} z^{n}$, be in the class $T_{p, q} R(\lambda, \alpha, \beta, \mu, \theta)$. For $t \in(0,1)$, it is enough to show that the function $h(z)=(1-t) f(z)+\operatorname{tg}(z)$ is in the class $T_{p, q} R(\lambda, \alpha, \beta, \mu, \theta)$. Since $h(z)=z^{p}-$ $\sum_{n=p+1}^{+\infty}\left((1-t) a_{n}+t b_{n}\right) z^{n}$

$\sum_{n=p+1}^{+\infty}[(n(n-1)-(\alpha+\beta)+1) \lambda+(n+1)(\alpha+\beta)-1] M(n, p, q, \mu, \theta)\left((1-t) a_{n}+t b_{n}\right) b_{n} \leq(1-2 \lambda)$

and so $h(z) \in T_{p, q} R(\lambda, \alpha, \beta, \mu, \theta)$.

Corollary 3.1. Let $f_{k}(z), 1 \leq k \leq m$, defined by $f_{k}(z)=z^{p}-\sum_{n=p+1}^{+\infty} a_{n, k} z^{n}$ be in the class $T_{p, q} R(\lambda, \alpha, \beta, \mu, \theta)$, then the function $F(z)=\sum_{k=1}^{m} c_{k} f_{k}(z)$ is also in $T_{p, q} R(\lambda, \alpha, \beta, \mu, \theta)$, where $\sum_{k=1}^{m} c_{k}=1$.

\section{Integral operators on $T_{p, q} R(\lambda, \alpha, \beta, \mu, \theta)$}

In this section we investigate properties of functions in the class $T_{p, q} R(\lambda, \alpha, \beta, \mu, \theta)$, involving the familiar operator $F_{c}(z)$.

Theorem 4.1. If $f(z)=z^{p}-\sum_{n=p+1}^{+\infty} a_{n} z^{n}$ belongs to $T_{p, q} R(\lambda, \alpha, \beta, \mu, \theta)$, then the function $F_{c}(z)$ defined by $F_{c}(z)=\frac{c+p}{z^{c}} \int_{0}^{1} t^{c} f(t z) d t, c \geq 1$, is also in $T_{p, q} R(\lambda, \alpha, \beta, \mu, \theta)$. 
Proof. Since $f(z)$ belong to $T_{p, q} R(\lambda, \alpha, \beta, \mu, \theta)$,

$$
\begin{aligned}
F_{c}(z) & =\frac{p+c}{z^{c}} \int_{0}^{z} t^{c-1}\left[z^{p}-\sum_{n=p+1}^{+\infty} a_{n} t^{n}\right] d t \quad, \quad c>1, \\
& =\frac{p+c}{z^{c}} \int_{0}^{z}\left[t^{p+c-1}-\sum_{n=p+1}^{+\infty} a_{n} t^{n+c-1}\right] d t \\
& =\frac{p+c}{z^{c}}\left[\frac{1}{p+c} t^{p+c}-\sum_{n=p+1}^{+\infty} a_{n} \frac{1}{n+c} t^{n+c}\right]_{0}^{z} \\
& =\frac{p+c}{z^{c}}\left[\frac{1}{p+c} z^{p+c}-\sum_{n=p+1}^{+\infty} a_{n} \frac{1}{n+c} z^{n+c}\right] \\
& =z^{p}-\sum_{n=p+1}^{+\infty} \frac{p+c}{n+c} a_{n} z^{n}
\end{aligned}
$$

Since $\frac{p+c}{n+c}<1$

$$
\begin{aligned}
& \sum_{n=p+1}^{+\infty} \frac{p+c}{n+c}[(n(n-1)-(\alpha+\beta)+1) \lambda+(n+1)(\alpha+\beta)-1] M(n, p, q, \mu, \theta) a_{n} b_{n} \\
\leq & \sum_{n=p+1}^{+\infty}[(n(n-1)-(\alpha+\beta)+1) \lambda+(n+1)(\alpha+\beta)-1] M(n, p, q, \mu, \theta) a_{n} b_{n} \\
\leq & (1-2 \lambda) .
\end{aligned}
$$

Hence the result.

Corollary 4.1. If $f(z) \in T_{p, q} R(\lambda, \alpha, \beta, \mu, \theta)$ and $F_{c}(z)$ is defined as $F_{c}(z)=$ $c \int_{0}^{1} v^{c} f(v z) d v, c \geq 1$. Then $F_{c}(z) \in T_{p, q} R(\lambda, \alpha, \beta, \mu, \theta)$.

\section{RE F E R E N C E S}

1. E. S. AqLan: Some problems connected with geometric function theory, Ph.D. Thesis, Pune University, 2004.

2. W. G. Atshan and R. H. Buti: Fractional calculus of a class of univalent functions with negative coefficients defined by Hadamard product with Rafid-operator, European J. Pure Appl. Math. 4(2) (2011), 162-173.

3. A. H. El-QAdeem and S. K. Al-Ghazal: On subclasse of p-valent functions with negative coefficients defined by using Rafid operator, Comput. Math. Appl. (2019). 
4. A. H. El-QADeem and S. K. Al-Ghazal: Some aspect of certain two subclass of analytic functions with negative coefficients defined by using Rafid operator, arXiv:1904.07913v1.

5. G. Gasper and M. Rahman: Basic Hypergeometric series, Cambridge University Press, Cambridge, 1990.

6. F. H. JACKSON: On q-functions and a certain difference operator, Trans. Royal Soc. Edinburgh, 46 (2) (1909), 253-281.

7. F. H. Jackson: On q-definite integrals, Q. J. Pure Appl. Math., 41 (1910), 193203.

8. F. H. JACKSON: q-Difference equations, Am. J. Math., 32 (1910), 305-314.

9. A. E. Livingston: On the radius of univalence of certain analytic functions, Proc. Amer. Math. Soc., 17 (1966), 99- 107.

10. J. L. LiU: The Noor integral and strongly starlike functions, J. Math. Anal. Appl. 261 (2001), 441-447.

11. R. J. LiberA: Some classes of regular univalent functions, Proc. Amer. Math. Soc., 16 (1969), 755-758.

12. Z. S. I. GASPER: Linear sequential q-difference equations of fractional order, Fract. Calc. Appl. Anal., 12 (2) (2009), 159-178.

13. A. O. Mostafa, H. M. Aouf and T. Bulboaca: Convolution conditions for subelasse of mermorphic functions of complex order associated with basic Bessel functions, J. Egyptian Math. Soc 25 (2017), 286-290.

14. T. M. SEOudy and M. K. Aouf: Convolution properties for certain classes of analytic functions defined by q-derivative operator, Abstr. Appl. Anal., 2014 (2014), Art. ID 846719, 1-7. 